\title{
ERLANGIAN APPROXIMATIONS \\ FOR FINITE-HORIZON RUIN PROBABILITIES
}

BY

Soren Asmussen', Florin Avram ${ }^{2}$ and Miguel Usabel ${ }^{3}$

\begin{abstract}
For the Cramér-Lundberg risk model with phase-type claims, it is shown that the probability of ruin before an independent phase-type time $H$ coincides with the ruin probability in a certain Markovian fluid model and therefore has an matrix-exponential form. When $H$ is exponential, this yields in particular a probabilistic interpretation of a recent result of Avram \& Usabel. When $H$ is Erlang, the matrix algebra takes a simple recursive form, and fixing the mean of $H$ at $T$ and letting the number of stages go to infinity yields a quick approximation procedure for the probability of ruin before time $T$. Numerical examples are given, including a combination with extrapolation.
\end{abstract}

\section{KEYWORDS}

Erlang expiration, extrapolation, finite time ruin probabilities, fluid model, phasetype distributions, semi-Markov embedding.

\section{INTRODUCTION}

Consider the reserve $R_{t}=u-S_{t}$ at time $t$ of an insurance company where $S$ is a Lévy process of the form

$$
S_{t}=\sum_{k=1}^{N_{t}} Z_{k}-c t
$$

$Z_{1}, Z_{2}, \ldots$ are i.i.d. random variables with common distribution $F$ concentrated on $(0, \infty)$ and $N$ is a Poisson processes with arrival rate $\lambda\left(N, Z_{1}, Z_{2}, \ldots\right.$ are independent). We will assume w.l.o.g. (by scaling time) that $c=1$.

1 Mathematical Statistics, Centre for Mathematical Sciences, Box 118, 22100 Lund, Sweden, E-mail: asmus@maths.lth.se.

2 Dept. de Math., Universite de Pau and Dept. of Actuarial Maths. \& Statistics, Heriot-Watt University, Edinburgh EH14 4AS U.K., E-mail: Florin.Avram@univ-Pau.fr.

3 Edif. Miguel de Unamuno, Universidad Carlos III, Avda. Universidad Carlos III 22, 28270 Colmenarejo (Madrid), Spain, E-mail: usabel@emp.uc3m.es. 
The time of ruin is

$$
\tau=\tau(u)=\inf \left\{t>0: R_{t}<0\right\}=\inf \left\{t>0: S_{t}>u\right\},
$$

the infinite horizon ruin probability is $\psi(u)=\mathbf{P}(\tau(u)<\infty)$ and the probability of ruin before time $T$ is

$$
\psi(u, T)=\mathbf{P}(\tau(u) \leq T)
$$

(the probability and expectation are taken with respect to the process $S_{t}$ ).

Typically, the study of $\psi(u, T)$ is much harder than that of $\psi(u)$, and even $\psi(u)$ can only be found explicitly in a few cases, mainly when $F$ is phase-type (see, e.g., [20], [21], [3], [17] or [7] for background and terminology). With $\alpha$ the initial row vector and $\boldsymbol{G}$ the phase generator, it then holds that

$$
\psi(u)=\eta e^{(G+g B f \eta) u} 1
$$

where $\boldsymbol{g}=-\boldsymbol{G} \mathbf{1}$ with $\mathbf{1}=(1 \ldots 1)^{\prime}$ and $\boldsymbol{\eta}=-(\lambda / c) \alpha G^{-1}$. This was first pointed out by Asmussen \& Rolski [10] in the risk theoretic setting, but in fact formula (2) is identical to the representation of the $\mathrm{M} / \mathrm{PH} / 1$ waiting time distribution given in Neuts [20]. In the finite horizon case, the phase-type assumption did so far not appear to provide any substantial simplication (but see Stanford \& Stroinski [23] for an attempt). However, recently Avram \& Usabel [11] found a formula of similar form as (2) for the probability of ruin before an exponential time $H_{a}$ independent of the risk process: if $H=H_{a}$ is exponential with rate $a$ and

$$
\psi_{a}(u)=\psi\left(u, H_{a}\right)=\mathbf{P}\left(\tau(u) \leq H_{a}\right)=\mathbf{E} e^{-a \tau(u)}
$$

(where the last equality holds by integration by parts), then

$$
\psi\left(u, H_{a}\right)=\boldsymbol{\eta}_{a} e^{\left(\boldsymbol{G}+g \eta_{a}\right) u} \mathbf{1}
$$

where $\boldsymbol{\eta}_{a}=(\lambda / c) \alpha\left(s_{a} \boldsymbol{I}-\boldsymbol{G}\right)^{-1}$ and $s_{a}$ is the unique positive root of the Cramér Lundberg equation (12). Their result dealt more generally also with the deficit at ruin, which is in fact always automatic for phase type jumps - see Corollary 4 . The approach was algebraic, with a key step in the proof being a linear algebra identity similar to that used in Asmussen \& Bladt [8].

In the present paper we generalize the results of [11] to a random horizon $H$ with a general phase-type distribution, using a different approach proposed in Asmussen [7]. This approach solves perpetual first time passage problems for Levy processes with phase-type jumps by embedding them first into an equivalent continuous Markov modulated diffusion with a finite order Markovian environment (also called fluid model in the absence of a Brownian component). This has the analytic advantage of replacing the original Markovian integrodifferential generator by a simpler first order ordinary-differential generator for the fluid model (see [13] and the proof of Theorem 2 below), and reducing 
ultimately (at least for constant premiums) this type of problems to obtaining the Jordan decomposition of certain matrices. The end result is that formulas like (2), (4), are available under a wide variety of models, like for example that of phase-type renewal arrivals [13]. Moreover, as shown below, we may also incorporate a phase-type horizon $H$ merely by increasing the dimension of the modulating environment (and of $\boldsymbol{\eta}$ ).

The case of an Erlang $H$ is of particular interest. Such an $H$ with a large number $L$ of stages provides a very good approximation for its mean since, as shown by Aldous \& Shepp [1], the Erlang distribution is the phase-type distribution with minimal variance for a given number of phases. Correspondingly, we find in Theorem 6 that if we fix the mean $T$ and let $L$ go to infinity, the corresponding $\psi(u, H)$ converges to $\psi(u, T)$ with a rate of convergence which is optimal. Furthermore, the Erlangian case turns out to be simpler computationally, since it leads to an explicit recursion for $\boldsymbol{U}$. We thereby provide a quick route to approximations of $\psi(u, T)$ (a topic which is in general not easy at all - see the survey in [7] Ch. IV). We illustrate the approach with a couple of numerical examples which show that indeed a good fit is obtained with relatively few stages $L$, and that the efficiency of the numerical scheme is much improved when combined with extrapolation.

\section{ThE PRobability of RUIN AT A PHASE-TYPE TIME}

The connection between Levy processes with phase-type jumps (or, more generally, Markov modulated Levy processes with phase-type jumps) and Markovmodulated diffusions follows from an observation which seems to have been first exploited in Asmussen [5] (see also [6], [7]) and Asmussen \& Kella [9]). The general trick is to level out the positive jumps to sample path segments with slope +1 and the negative jumps to sample path segments with slope -1 and add an extra phase say $\zeta$ (or more if necessary), for the "regular time" when the process drifts. This embeds the process with phase-type jumps $S_{t}$ in a continuous Markov additive process $(J, V)$, where the Markov component $J_{t}$ (called environment) is $\zeta$ at a regular time and gives the current phase of the jump otherwise.

In this way, one obtains a semi-Markov (or Markov modulated) risk process of the kind studied in [2], but much simpler, in that there are no jumps, but only Markov modulated deterministic drifts (or "premiums"). This approach has technical advantages, like removing certain integrability difficulties arising due to the jumps (see Asmussen \& Kella [9] Theorem 2.1.4); more importantly, it replaces the original problem involving an integro-differential equation by a related simpler problem involving a first order differential system for the resulting fluid model.

In our case of a phase-type horizon $H$, we have to keep track also of the phase of the horizon, and therefore the imbedding of $S_{t}$ in a Markovian fluid $\left\{\left(J_{t}, V_{t}\right)\right\}$ is slightly more complicated than the one described before. Assume that the set of phases for the jump distribution $F$ is $E_{F}$ with $p_{F}$ elements and that $H$ is phase-type with set of phases $E_{H}$ with $p_{H}$ elements, phase generator $\boldsymbol{H}$ 
and initial vector $\boldsymbol{\beta}$. We denote by $\boldsymbol{h}=-\boldsymbol{H} \mathbf{1}$ the rates of absorbtion of the phase process into its absorption "cemetery" state $C$ (note that $\mathbf{1}$ and similarly the identity matrix $I$ and zero vectors/matrices 0 will have a dimension varying on the context throughout the paper). The background Markov process $\left\{J_{t}\right\}$ has state space $E=E_{0} \cup E_{+} \times E_{-}$where $E_{0}=\{C\}$ is the absorbing state, $E_{-}=E_{H}$, $E_{+}=E_{H} \times E_{F}$ (matrices and vectors are written in block-partitioned form corresponding to this ordering, and we write $\left.p_{-}=p_{H}, p_{+}=p_{H}+p_{F}\right)$. The fluid model $\left\{V_{t}\right\}$ moves linearly at a rate $r_{i}$ when $J_{t}=i$; more precisely, it is defined as follows:

- $J_{t}=C$ corresponds to the final segment of the risk process after time $H$ and here $r_{C}=0$.

- $J_{t} \in E_{-}=E_{F}$ corresponds to a segment of the risk process $S_{t}$ without jumps, i.e., downwards motion at the linear rate $r_{i}=-1$ and the state for $\left\{J_{t}\right\}$ is the current phase of the phase process for $H$.

- $J_{t} \in E_{+}=E_{H} \times E_{F}$ corresponds to a jump of the risk process. The first component of $\left\{J_{t}\right\}$ is fixed at the current phase of the phase process for $H$ during a segment corresponding to a fixed jump, and the second goes through the phases of the phase process for the jump. Further, $r_{i}=1$ for $i$ $\in E_{H} \times E_{F}$.

It follows that the initial distribution for $\left\{J_{t}\right\}$ is $(0 \boldsymbol{\beta} \mathbf{0})$ and that its transitions intensity matrix is

$$
\boldsymbol{\Lambda}=\left(\begin{array}{ccc}
0 & 0 & 0 \\
\boldsymbol{h} & \boldsymbol{H}-\lambda \boldsymbol{I} & \lambda \boldsymbol{I} \otimes \boldsymbol{\alpha} \\
\mathbf{0} & \boldsymbol{I} \otimes \boldsymbol{g} & \boldsymbol{I} \otimes \boldsymbol{G}
\end{array}\right)=\left(\begin{array}{ccc}
0 & \mathbf{0} & \mathbf{0} \\
\boldsymbol{h} & \boldsymbol{\Lambda}^{--} & \boldsymbol{\Lambda}^{++} \\
\mathbf{0} & \boldsymbol{\Lambda}^{+-} & \boldsymbol{\Lambda}^{++}
\end{array}\right)
$$

(we used notation like $\Lambda^{+-}=\boldsymbol{I} \otimes g, \ldots$; for Kronecker products $\otimes$, see e.g. Graham [16]. For example, if $H$ is exponential with rate $a$, we have

$$
\boldsymbol{\Lambda}=\left(\begin{array}{ccc}
0 & 0 & 0 \\
a & -a-\lambda & \lambda \boldsymbol{\alpha} \\
\mathbf{0} & \boldsymbol{g} & \boldsymbol{G}
\end{array}\right)
$$

and if $H$ is Erlang(2) with rate $a$, then

$$
\boldsymbol{\Lambda}=\left(\begin{array}{c|cc|cc}
0 & 0 & 0 & \mathbf{0} & \mathbf{0} \\
\hline 0 & -a-\lambda & a & \lambda \boldsymbol{\alpha} & \mathbf{0} \\
a & 0 & -a-\lambda & \mathbf{0} & \lambda \boldsymbol{\alpha} \\
\hline \mathbf{0} & \boldsymbol{g} & \mathbf{0} & \boldsymbol{G} & \mathbf{0} \\
\mathbf{0} & \mathbf{0} & \boldsymbol{g} & \mathbf{0} & \boldsymbol{G}
\end{array}\right)
$$




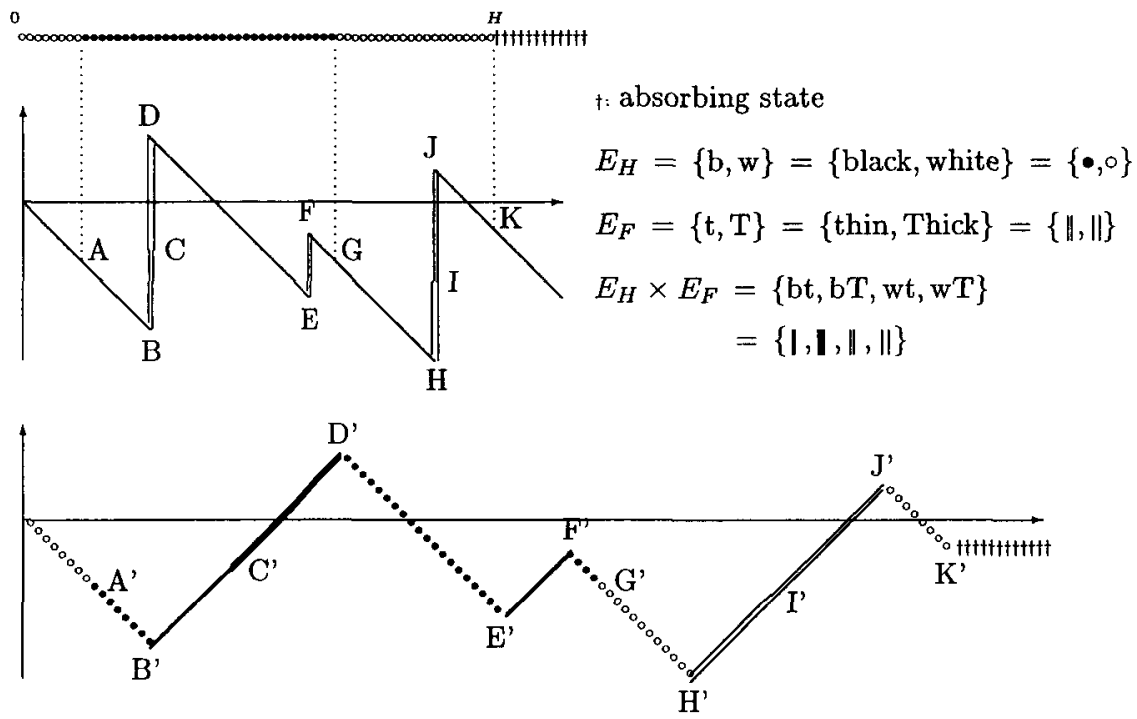

Figure 1

Fig. 1 illustrates the connection between $S_{t}$ (upper part) and $\left(J_{t}, V_{t}\right)$ (lower part). The following result follows immediately by a sample path comparison.

Theorem 1. $\psi(u, H)$ coincides with the infinite horizon ruin probability for $\left\{V_{t}\right\}$ (i.e. with the probability that $\left\{V_{t}\right\}$ will ever exceed level $u$ ).

We state now a general result concerning the upcrossing probabilities of fluid models due to [14] and [18] and generalized by [4] to the case of Markov-modulated diffusions, following Theorem 3.2 of [4]. Here, the special structure of the matrices $\mathbf{\Lambda}^{--}$etc plays no role; we will see however in the next section that if $H$ is Erlang, then a considerably simpler recursive scheme is available.

Theorem 2. Let $\boldsymbol{\eta}$ denote the $p_{-} \times p_{+}$matrix of "upcrossing phase probabilities" at the completion of a downwards excursion for a fluid model $\left\{V_{t}\right\}$, conditioned on starting in a decreasing state $i \in E_{-}$, let $\psi(u)=\left(\psi_{i}(u), i=1,,,, p_{-}\right)$denote the infinite horizon phase-distribution at $u$ (starting at 0$)$, conditioned on starting in a decreasing state, and let $\boldsymbol{\phi}(u)=\left(\psi_{i}(u), i=1,,, p_{+}\right)$denote the infinite horizon phase-distribution at $u$, conditioned on starting in an increasing state. Then,

a) $\boldsymbol{\eta}$ satisfies the Riccati equation:

$$
\eta U+\Lambda^{--} \eta+\Lambda^{-+}=0
$$

and

$$
\psi(u)=\boldsymbol{\eta} \phi(u)=\eta e^{U_{u}} \mathbf{1}
$$


where $\boldsymbol{U}$ is the $p_{+} \times p_{+}$matrix

$$
\boldsymbol{U}=\boldsymbol{\Lambda}^{++}+\boldsymbol{\Lambda}^{+-} \boldsymbol{\eta}
$$

b) Let now $\delta>0$ be a "convergence improving parameter", satisfying $\delta>-h_{i i}+\lambda$ for all $i \in E_{H}$. Then, the solution $\eta$ of the Riccati equation (5) can be computed by the iteration scheme $\boldsymbol{\eta}=\lim _{n \rightarrow \infty} \boldsymbol{\eta}^{(n)}$ where $\boldsymbol{\eta}^{(0)}=0$,

$$
\boldsymbol{\eta}^{(n+1)}=\left(\left(\delta \boldsymbol{I}+\boldsymbol{\Lambda}^{--}\right) \boldsymbol{\eta}^{(n)}+\boldsymbol{\Lambda}^{-+}\right)\left(\delta \boldsymbol{I}-\left(\boldsymbol{\Lambda}^{++}+\boldsymbol{\Lambda}^{+-} \boldsymbol{\eta}^{(n)}\right)^{-1}\right.
$$

c) Alternatively, let $\boldsymbol{K}[s]$ be the matrix of dimension $p_{-}+p_{+}$given by

$$
\boldsymbol{K}[s]=\left(\begin{array}{cc}
\boldsymbol{\Lambda}^{--}-s \boldsymbol{I} & \boldsymbol{\Lambda}^{++} \\
\boldsymbol{\Lambda}^{+-} & \boldsymbol{\Lambda}^{++}+s \boldsymbol{I}
\end{array}\right)=\left(\begin{array}{cc}
\boldsymbol{H}-\lambda \boldsymbol{I}-s \boldsymbol{I} & \lambda \boldsymbol{I} \otimes \boldsymbol{\alpha} \\
\boldsymbol{I} \otimes \boldsymbol{g} & \boldsymbol{I} \otimes \boldsymbol{G}+s \boldsymbol{I}
\end{array}\right)
$$

and assume that the equation $\boldsymbol{K}[s]=0$ has $p_{+}$distinct generalized eigenvalues $s_{1}$, $\ldots, s_{p_{+}}$with $\mathscr{R}\left(s_{i}\right)<0$ and let $\boldsymbol{k}_{\boldsymbol{i}}=\left(\boldsymbol{k}_{\boldsymbol{i}}^{(-)} \boldsymbol{k}_{i}^{(+)}\right), i=1, \ldots, p_{+}$be right (generalized) eigenvectors of $\boldsymbol{K}\left[s_{i}\right]$ corresponding to the eigenvalue $s_{i}$. Then $\boldsymbol{\eta}$ is the solution of the linear equations

$$
\boldsymbol{\eta}\left(\boldsymbol{k}_{1}^{(+)} \ldots \boldsymbol{k}_{p_{+}}^{(+)}\right)=\left(\boldsymbol{k}_{1}^{(-)} \ldots \boldsymbol{k}_{p_{+}}^{(-)}\right)
$$

and further

$$
\boldsymbol{U}=\left(s_{1} \boldsymbol{k}_{1}^{(+)} \ldots s_{p_{+}} \boldsymbol{k}_{p_{+}}^{(+)}\right)=\left(\boldsymbol{k}_{1}^{(+)} \ldots \boldsymbol{k}_{p_{+}}^{(+)}\right)^{-1}
$$

Proof. a,b) This is a reformulation of Theorem 3.2 of Asmussen [4], with the following small amendments. First, it is assumed there that $E_{0}=\emptyset$, but the separate treatment in [4] of the case $E_{0} \neq \emptyset$ is not needed here because $E_{0}$ is absorbing. Second, [4] gives

$$
\boldsymbol{\eta}=\left(\left(\delta \boldsymbol{I}+\boldsymbol{\Lambda}^{--}\right) \boldsymbol{\eta}+\boldsymbol{\Lambda}^{-+}\right)\left(\delta \boldsymbol{I}-\left(\boldsymbol{\Lambda}^{++}+\boldsymbol{\Lambda}^{+-} \boldsymbol{\eta}\right)^{-1}\right.
$$

from which (5) is obtained by multiplying by $\delta \boldsymbol{I}-\boldsymbol{U}$ to the right and eliminating $\delta$.

c) The proof is an immediate application of Section 5 of [4]. Note that the fact that (8) satisfies the Riccati equation for any choice of eigenvectors $\boldsymbol{k}_{i}$, $i=1, \ldots, p_{+}$is easily checked, but the fact that we need the negative real part eigenvalues requires some probabilistic argument - see also [9] for an optimal stopping approach.

QED

Notes: 1) Here is a brief outline of some of the ideas behind the proof of Theorem 2: Fixing the barrier at 0 and letting the starting point $u$ vary, we find that $\psi(u), \boldsymbol{\phi}(u)$ satisfy the Feynman-Kac equation:

$$
\left(\begin{array}{l|l}
\boldsymbol{I} & \mathbf{0} \\
\hline \mathbf{0} & -\boldsymbol{I}
\end{array}\right)\left(\begin{array}{l}
\boldsymbol{\psi}^{\prime}(u) \\
\boldsymbol{\phi}^{\prime}(u)
\end{array}\right)+\left(\begin{array}{c|c}
\boldsymbol{\Lambda}^{--} & \boldsymbol{\Lambda}^{-+} \\
\hline \boldsymbol{\Lambda}^{+-} & \boldsymbol{\Lambda}^{++}
\end{array}\right)\left(\begin{array}{l}
\boldsymbol{\psi}(u) \\
\boldsymbol{\phi}(u)
\end{array}\right)=0,\left(\begin{array}{l}
\boldsymbol{\psi}(\infty) \\
\boldsymbol{\phi}(0)
\end{array}\right)=\left(\begin{array}{l}
\mathbf{0} \\
1
\end{array}\right)
$$


or

$$
\begin{array}{ll}
\psi^{\prime}(u)=-\Lambda^{--} \psi(u)-\Lambda^{-+} \phi(u) & \psi(\infty)=0 \\
\phi^{\prime}(u)=\Lambda^{+-} \psi(u)+\Lambda^{++} \phi(u) & \phi(0)=\mathbf{1}
\end{array}
$$

Substituting now the (probabilistically obvious) first equality in (6) $\psi(u)=\eta \phi(u)$ transforms the second equation to the homogeneous form:

$$
\phi^{\prime}(u)=U \phi(u)
$$

where $U=\Lambda^{++}+\Lambda^{+-} \eta$, and thus $\psi(u)=e^{U u} 1$.

Also, this substitution transforms the first equation (after factoring $\phi(u)$ ) into the algebraic Riccati equation (5).

2) It is interesting to investigate, in the spirit of [8], whether this result holds also under the less restrictive assumption of jumps with a rational Laplace transform.

Finally, using the fact that in our case the horizon phases start with probabilities $\boldsymbol{\beta}$, we get:

Corollary 3. One has $\psi(u, H)=\boldsymbol{\beta} \eta e^{U} u_{1}$ where $\boldsymbol{\eta}$ is a $E_{-} \times E_{+}$matrix and $\boldsymbol{U} a$ $E_{+} \times E_{+}$matrix such that

$$
\begin{aligned}
& \boldsymbol{U}=\boldsymbol{I} \otimes \boldsymbol{G}+(\boldsymbol{I} \otimes \boldsymbol{g}) \boldsymbol{\eta}, \\
& \mathbf{0}=\boldsymbol{\eta} \boldsymbol{U}+\boldsymbol{H} \boldsymbol{\eta}-\lambda \boldsymbol{\eta}+\lambda \boldsymbol{I} \otimes \alpha .
\end{aligned}
$$

The deficit at the time of ruin $Y=S_{\tau}$ has also been studied by many authors, see e.g. [15]. An important advantage of phase-type modeling is that it renders the distribution of the deficit at ruin automatically. Indeed, it is easy to see from the probabilistic interpretation of Theorem 2 that this deficit is phasetype on $E_{+}$with initial vector $\beta \eta e^{U u}$ and phase generator $I \otimes G$, which yields the corollary:

Corollary 4. Let

$$
\psi(u, H, Y)=\mathbf{P}_{u}\{\tau<H ; Y>y\}
$$

denote the "finite time bivariate probability of ruin" with time span $H$ and severity of ruin bigger than $y$. Then,

$$
\psi(u, H, Y)=\boldsymbol{\beta} \eta e^{U u} e^{G y} \mathbf{1}
$$

\section{The Erlang Case}

We first verify that Theorem 2 gives the result of [11] for the exponential case. Here $E_{-}$consists of a single point so that $\eta$ is a $E_{+}=E_{F}$ row vector and $U$ a $E_{F}$ $\times E_{F}$ matrix, and (9), (10) take the form $\boldsymbol{U}=\boldsymbol{G}+\boldsymbol{g \eta}$,

$$
\mathbf{0}=\boldsymbol{\eta} \boldsymbol{U}-(a+\lambda) \boldsymbol{\eta}+\lambda \alpha=\boldsymbol{\eta} \boldsymbol{G}+\boldsymbol{\eta} \boldsymbol{\eta} \boldsymbol{\eta}-(a+\lambda) \boldsymbol{\eta}+\lambda \alpha .
$$


Defining $s=a+\lambda-\boldsymbol{\eta}$, this means $\mathbf{0}=\boldsymbol{\eta} \boldsymbol{G}-s \boldsymbol{\eta}+\lambda \alpha$ so that $\boldsymbol{\eta}=\lambda \boldsymbol{\alpha}(\boldsymbol{s} \boldsymbol{I}-\boldsymbol{G})^{-1}$. It only remains to compute $s$ but multiplying by $g$ to the right and appealing to the standard formula for the m.g.f. $f^{*}(s)$ of a phase -type distribution $F$, we get

$$
-a+s-\lambda+\lambda f^{*}(s)=\kappa(s)-a=0
$$

where $\kappa(s)=\log \mathbf{E} e^{s\left(R_{1}-u\right)}=s+\lambda\left(f^{*}(s)-1\right)$ denotes the Levy exponent of the process $R_{t}$. We note now, as in [11], that since the Laplace transform of the ruin function must be well defined over the positive numbers, $s$ must be the unique positive root of this equation ${ }^{4}$.

Next consider the Erlang(2) case. The probabilistic interpretation of $\boldsymbol{\eta}$ given in [4] is that $\eta_{i j}$ is the probability that when $J_{0}=i \in E_{-}$, then the first upcrossing of level 0 of the fluid model will occur at a time $t$ with $J_{t}=j \in E_{+}$. Taking into account the specific structure of the Erlang case, it follows that we can write

$$
\boldsymbol{\eta}=\left(\begin{array}{ll}
\boldsymbol{\eta}_{1} & \boldsymbol{\eta}_{2} \\
0 & \boldsymbol{\eta}_{1}
\end{array}\right)
$$

where $\boldsymbol{\eta}_{1}$ coincides with the $\boldsymbol{\eta}=\lambda \alpha(s \boldsymbol{I}-\boldsymbol{G})^{-1}$ just computed for the exponential case. By (9), (10)

$$
\begin{aligned}
\left(\begin{array}{ll}
\mathbf{0} & 0 \\
\mathbf{0} & \mathbf{0}
\end{array}\right)= & \left(\begin{array}{ll}
\boldsymbol{\eta}_{1} & \boldsymbol{\eta}_{2} \\
\mathbf{0} & \boldsymbol{\eta}_{1}
\end{array}\right)\left[\left(\begin{array}{cc}
\boldsymbol{G} & \mathbf{0} \\
\mathbf{0} & \boldsymbol{G}
\end{array}\right)+\left(\begin{array}{ll}
\boldsymbol{g} & \mathbf{0} \\
\mathbf{0} & \boldsymbol{g}
\end{array}\right)\left(\begin{array}{cc}
\boldsymbol{\eta}_{1} & \boldsymbol{\eta}_{2} \\
\mathbf{0} & \boldsymbol{\eta}_{1}
\end{array}\right)\right]+ \\
& \left(\begin{array}{cc}
-\lambda-a & a \\
0 & -\lambda-a
\end{array}\right)\left(\begin{array}{cc}
\boldsymbol{\eta}_{1} & \boldsymbol{\eta}_{2} \\
\mathbf{0} & \boldsymbol{\eta}_{1}
\end{array}\right)+\left(\begin{array}{cc}
\lambda \boldsymbol{\alpha} & \mathbf{0} \\
\mathbf{0} & \lambda \boldsymbol{\alpha}
\end{array}\right) .
\end{aligned}
$$

Entry by entry (in lexicographical order) this means

$$
\begin{aligned}
& 0=\boldsymbol{\eta}_{1} \boldsymbol{G}+\boldsymbol{\eta}_{1} \boldsymbol{g} \boldsymbol{\eta}_{1}-(\lambda+a) \boldsymbol{\eta}_{1}+\lambda \alpha \\
& 0=\boldsymbol{\eta}_{2} \boldsymbol{G}+\boldsymbol{\eta}_{1} \boldsymbol{g} \boldsymbol{\eta}_{2}+\boldsymbol{\eta}_{2} \boldsymbol{g} \boldsymbol{\eta}_{1}-(\lambda+a) \boldsymbol{\eta}_{2}+a \boldsymbol{\eta}_{1} \\
& 0=\mathbf{0} \\
& 0=\boldsymbol{\eta}_{1} \boldsymbol{G}+\boldsymbol{\eta}_{1} \boldsymbol{g} \boldsymbol{\eta}_{1}-(\lambda+a) \boldsymbol{\eta}_{1}+\lambda \alpha
\end{aligned}
$$

It is seen that only the second equation contains information on $\boldsymbol{\eta}_{\mathbf{2}}$, and recalling that $\eta_{1} g=\lambda+a-s$, it yields

$$
\eta_{2}=a \eta_{1}\left(s I-G-g \eta_{1}\right)^{-1} .
$$

4 To see that for $a>0$ the equation $\kappa(\theta)-a=0$ has always exactly one positive solution, note that since the Levy exponent $\kappa$ is convex over $\mathbb{R}^{+}$, the equation may have at most 2 nonegative solutions. Furthemore, when $a=0, \theta=0$ is always a solution; let $\theta_{0}$ denote the largest solution in this case. Note that $\theta_{0}>0$ if and only if $\kappa^{\prime}(0)=\mathbf{E}\left(R_{1}\right)<0$. Also, $\kappa(\theta) \leq 0$ for $\theta \in\left[0, \theta_{0}\right]$ while $\kappa$ is increasing over the interval $\left[\theta_{0}, \infty\right)$; therefore, $\kappa(\theta)$ has a unique continuous inverse $\gamma(a) \geq \theta_{0}$ which is defined for $a \geq 0$; therefore, $\kappa(\theta)-a=0$ has always a unique positive solution. 
In the general case:

Theorem 5. Assume that $H$ is Erlang with L stages. Then $\eta$ is an upper triangular block Toeplitz matrix, with entries $\boldsymbol{\eta}_{1}, \boldsymbol{\eta}_{2}, \ldots, \boldsymbol{\eta}_{L}$ given by the recursion

$$
\boldsymbol{\eta}_{L}=\left(a \boldsymbol{\eta}_{L-1}+\sum_{j=2}^{L-1} \boldsymbol{\eta}_{L-j+1} g \boldsymbol{\eta}_{j}\right)\left(s \boldsymbol{I}-\boldsymbol{G}-\boldsymbol{g} \boldsymbol{\eta}_{1}\right)^{-1}
$$

and $\boldsymbol{\eta}_{1}=\lambda \alpha(s \boldsymbol{I}-\boldsymbol{G})^{-1}$. In particular, $\boldsymbol{\eta}_{\mathbf{2}}$ is given by (13) and

$$
\begin{aligned}
& \boldsymbol{\eta}_{3}=\left(a \boldsymbol{\eta}_{2}+\boldsymbol{\eta}_{2} \boldsymbol{g} \boldsymbol{\eta}_{2}\right)\left(\boldsymbol{s} \boldsymbol{I}-\boldsymbol{G}-\boldsymbol{g} \boldsymbol{\eta}_{1}\right)^{-1}, \\
& \boldsymbol{\eta}_{4}=\left(a \boldsymbol{\eta}_{3}+\boldsymbol{\eta}_{3} \boldsymbol{g} \boldsymbol{\eta}_{2}+\boldsymbol{\eta}_{2} \boldsymbol{g} \boldsymbol{\eta}_{3}\right)\left(\boldsymbol{s} \boldsymbol{I}-\boldsymbol{G}-\boldsymbol{g} \boldsymbol{\eta}_{1}\right)^{-1}
\end{aligned}
$$

Proof. The form of $\boldsymbol{\eta}$ is seen exactly as when $L=2$. Considering the upper right block, (9), (10) yield

$$
0=\boldsymbol{\eta}_{L} \boldsymbol{G}+\sum_{j=1}^{L} \boldsymbol{\eta}_{L-j+1} \boldsymbol{g} \boldsymbol{\eta}_{j}-(\lambda+a) \boldsymbol{\eta}_{L-1},
$$

and from this (14) follows.

Theorem 6. Let $T>0$ be a fixed time and let $H_{L}$ denote the Erlang distribution with $L$ stages and mean $T$ (i.e., $a=L / T)$. Then $\psi\left(u, H_{L}\right) \rightarrow \psi(u, T)$ as $L \rightarrow \infty$. More precisely, for some constant $D$

$$
\psi\left(u, H_{L}\right)=\psi(u, T)+\frac{D}{L}+O\left(L^{-2}\right)
$$

Proof. Let $0<\epsilon<T$. Then it is immediately checked that for all $k, \mathbf{E}\left[H_{L}^{k}\right.$; $\left.\left|H_{L}-T\right|\right]>\epsilon$ is of order say $O\left(e^{-\epsilon_{\kappa} L}\right)$ (with $\epsilon_{\kappa}>0$ ) as $L \rightarrow \infty$. Since it is readily checked that the $k$ th derivative $\psi^{(\kappa)}(u, t)$ of $\psi(u, t)$ w.r.t. $t$ exists and is continuous, in particular bounded on $[T-\epsilon, T+\epsilon]$, it follows (using $\mathbf{E}\left(H_{L}-T\right)^{4}=$ $\left.3 / L^{2}+6 i L^{3}\right)$ that

$$
\begin{aligned}
\psi\left(u, H_{L}\right)= & \mathbf{P}\left(\tau(u)<H_{L} ; H_{L} \in[T-\epsilon, T+\epsilon]\right)+O\left(e^{-\epsilon_{0} L}\right) \\
= & \psi(u, T)+\sum_{k=1}^{3} \mathbf{E}\left[\frac{\left(H_{L}-T\right)^{k}}{k !} ; H_{L} \in[T-\epsilon, T+\epsilon]\right] \psi^{(k)}(u, T) \\
& +O\left(E\left(H_{L}-T\right)^{4}\right)+O\left(e^{-\epsilon_{0} L}\right) \\
= & \psi(u, T)+\sum_{k=1}^{3} \psi^{(k)}(u, T) \frac{\mathbf{E}\left(H_{L}-T\right)^{k}}{k !}+O\left(3 / L^{2}+6 / L^{3}\right) \\
= & \psi(u, T)+\psi^{(2)}(u, T) \frac{1 / L}{2 !}+\psi^{(3)}(u, T) \frac{2 / L^{2}}{3 !}+O\left(L^{-2}\right)
\end{aligned}
$$




\section{NUMERICAL EXAMPLES}

We implement now the Erlang method in practical calculations of ruin probabilities using the phase-type formula $\psi(u, H)=\left(\boldsymbol{\eta}_{1}, \boldsymbol{\eta}_{2}, \ldots, \boldsymbol{\eta}_{L}\right) e^{U u} \mathbf{1}$ and the simple recursive algorithm presented in the former section. The density of the claim sizes is $f(x)=\alpha e^{G x} g$ and the relative security loading considered in the illustrations below is $\theta=0.1$. In order to compare the Erlang approximations with the exact values $(L=\infty)$ of the ruin probabilities, the Gaver-Stehfest method of inverting the Laplace transform was used, see, for instance, Usabel [24].

We will first consider the hyperexponential distribution used by Wikstad [25] as an example of a highly skewed distribution; in matrix notation

$$
\begin{aligned}
\alpha_{1} & =\left(\begin{array}{ccc}
0.0039793 & 0.1078392 & 0.8881815
\end{array}\right) \\
\boldsymbol{G}_{1} & =\left(\begin{array}{ccc}
-0.014631 & 0 & 0 \\
0 & -0.190206 & 0 \\
0 & 0 & -5.514588
\end{array}\right)
\end{aligned}
$$

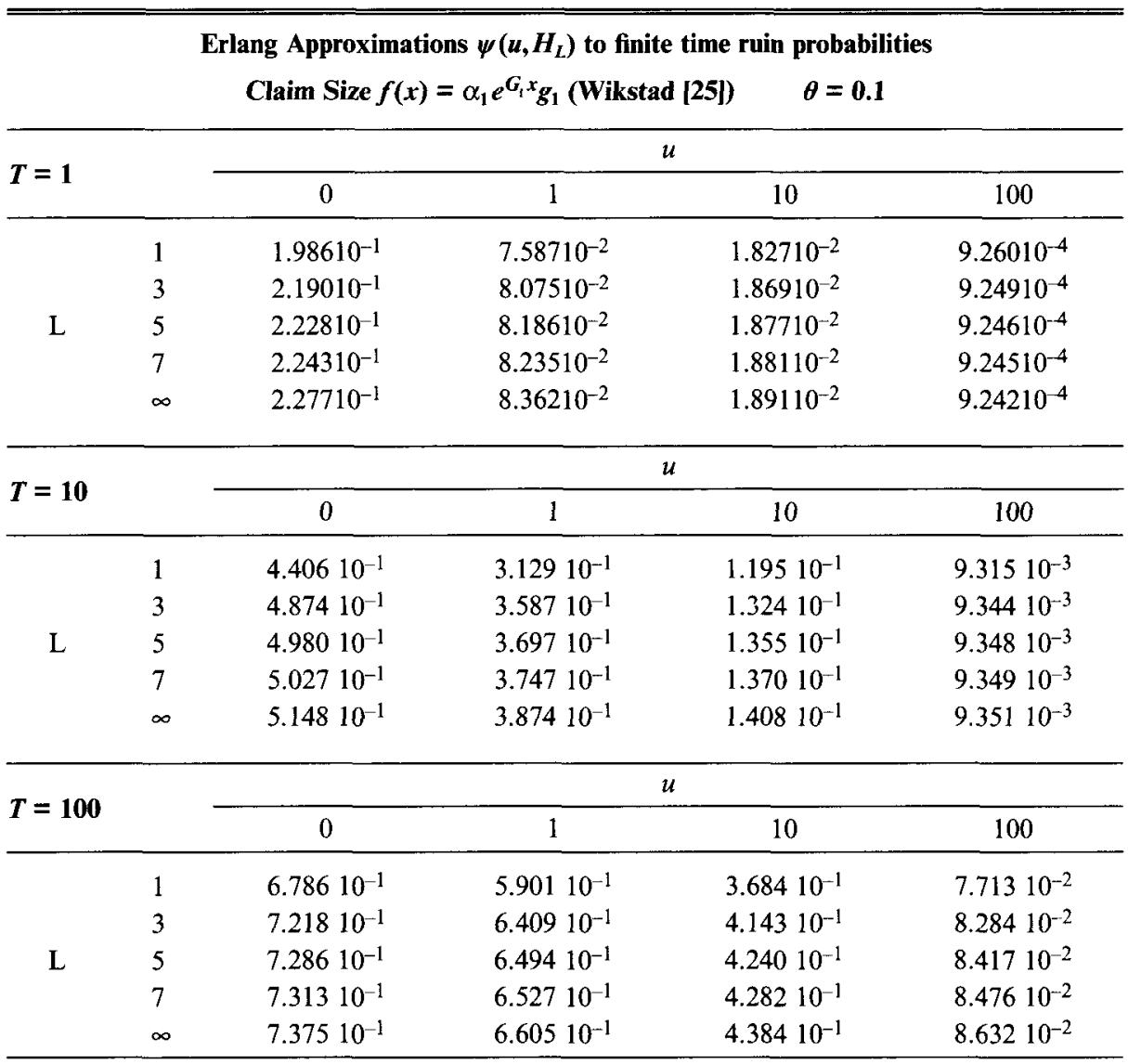




\begin{tabular}{|c|c|c|c|c|c|}
\hline \multirow{2}{*}{\multicolumn{2}{|c|}{$T=1000$}} & \multicolumn{4}{|c|}{$u$} \\
\hline & & 0 & 1 & 10 & 100 \\
\hline \multirow{5}{*}{ L } & 1 & $8.29010^{-1}$ & $7.79210^{-1}$ & $6.33910^{-1}$ & $2.92210^{-1}$ \\
\hline & 3 & $8.56910^{-1}$ & $8.14610^{-1}$ & $6.86010^{-1}$ & $3.35710^{-1}$ \\
\hline & 5 & $8.61310^{-1}$ & $8.20310^{-1}$ & $6.95310^{-1}$ & $3.45910^{-1}$ \\
\hline & 7 & $8.63110^{-1}$ & $8.22610^{-1}$ & $6.99010^{-1}$ & $3.50410^{-1}$ \\
\hline & $\infty$ & $8.67210^{-1}$ & $8.27810^{-1}$ & $7.07710^{-1}$ & $3.61810^{-1}$ \\
\hline
\end{tabular}

Next we consider a more regular claim size distribution, an Erlang(3) with mean 1 , in matrix notation

$$
\begin{aligned}
& \boldsymbol{\alpha}_{2}=\left(\begin{array}{lll}
1 & 0 & 0
\end{array}\right) \\
& \boldsymbol{G}_{2}=\left(\begin{array}{ccc}
-3 & 3 & 0 \\
0 & -3 & 3 \\
0 & 0 & -3
\end{array}\right)
\end{aligned}
$$

\begin{tabular}{|c|c|c|c|c|}
\hline \multicolumn{5}{|c|}{ Erlang Approximations $\psi\left(u, H_{L}\right)$ to finite time ruin probabilities } \\
\hline & & \multicolumn{2}{|c|}{ Claim Size $f(x)=\alpha_{2} e^{G_{2} x} g_{2}($ Erlang(3)) } & \multirow[t]{2}{*}{$\theta=0.1$} \\
\hline \multirow{2}{*}{$T=1$} & & \multicolumn{2}{|r|}{$u$} & \\
\hline & & 0 & 1 & 10 \\
\hline \multirow{5}{*}{$\mathrm{L}$} & 1 & $4.16910^{-1}$ & $2.08110^{-1}$ & $5.15910^{-5}$ \\
\hline & 3 & $4.85310^{-1}$ & $2.33910^{-1}$ & $1.32410^{-5}$ \\
\hline & 5 & $5.02910^{-1}$ & $2.40310^{-1}$ & $8.34810^{-6}$ \\
\hline & 7 & $5.10910^{-1}$ & $2.43210^{-1}$ & $6.59410^{-6}$ \\
\hline & $\infty$ & $5.32310^{-1}$ & $2.50810^{-1}$ & $3.14610^{-6}$ \\
\hline \multirow{2}{*}{$T=10$} & & \multicolumn{3}{|c|}{$u$} \\
\hline & & 0 & 1 & 10 \\
\hline \multirow{5}{*}{$\mathrm{L}$} & 1 & $7.35010^{-1}$ & $5.20610^{-1}$ & $1.35510^{-2}$ \\
\hline & 3 & $7.94910^{-1}$ & $5.83710^{-1}$ & $1.11210^{-2}$ \\
\hline & 5 & $8.03710^{-1}$ & $5.96310^{-1}$ & $1.03310^{-2}$ \\
\hline & 7 & $8.07110^{-1}$ & $6.01610^{-1}$ & $9.94310^{-3}$ \\
\hline & $\infty$ & $8.14810^{-1}$ & $6.14210^{-1}$ & $8.79710^{-3}$ \\
\hline \multirow{2}{*}{$T=100$} & & \multicolumn{3}{|c|}{$u$} \\
\hline & & 0 & 1 & 10 \\
\hline \multirow{5}{*}{$\mathrm{L}$} & 1 & $8.68710^{-1}$ & $7.28910^{-1}$ & $1.20310^{-1}$ \\
\hline & 3 & $8.91510^{-1}$ & $7.67510^{-1}$ & $1.43910^{-1}$ \\
\hline & 5 & $8.94110^{-1}$ & $7.72810^{-1}$ & $1.50210^{-1}$ \\
\hline & 7 & $8.95110^{-1}$ & $7.74810^{-1}$ & $1.53210^{-1}$ \\
\hline & $\infty$ & $8.97310^{-1}$ & $7.79310^{-1}$ & $1.60810^{-1}$ \\
\hline
\end{tabular}




\begin{tabular}{cccccc}
\hline \multirow{2}{*}{$\boldsymbol{T}=\mathbf{1 0 0 0}$} & \multicolumn{5}{c}{$u$} \\
\cline { 2 - 6 } & & 0 & 1 & 10 & 100 \\
\hline \multirow{4}{*}{$\mathrm{L}$} & 1 & $9.03410^{-1}$ & $7.93410^{-1}$ & $2.11010^{-1}$ & $3.72110^{-7}$ \\
& 3 & $9.08610^{-1}$ & $8.03310^{-1}$ & $2.27910^{-1}$ & $4.51010^{-7}$ \\
& 5 & $9.08910^{-1}$ & $8.03910^{-1}$ & $2.29610^{-1}$ & $4.81310^{-7}$ \\
& 7 & $9.08910^{-1}$ & $8.04110^{-1}$ & $2.30210^{-1}$ & $4.98910^{-7}$ \\
& $\infty$ & $9.09110^{-1}$ & $8.04310^{-1}$ & $2.31010^{-1}$ & $5.73710^{-7}$ \\
\hline \hline
\end{tabular}

The reader can see at a glance how quickly the approximations yield at least one or two significant digits even for very small values of the ruin probabilities. Furthermore, motivated by the linear rate of convergence (15), we implemented next the Richardson extrapolation (see e.g. [22]), leading to the improved estimate

$$
\psi_{1}(u, T) \approx(L+1) \psi\left(u, H_{L+1}\right)-L \psi\left(u, H_{L}\right)
$$

with error going to 0 at rate $1 / L^{2}$.

Improved Erlang Approximations $\psi_{1}\left(u, H_{L}\right)$ to finite time ruin probabilities

Claim Size $f(x)=\alpha_{1} e^{G_{1} x} g_{1}($ Wikstad(1971)) $\quad \theta=0.1$

\begin{tabular}{cccccc}
\hline \multirow{T}{*}{$=\mathbf{1}$} & \multicolumn{5}{c}{$u$} \\
\cline { 3 - 6 } & & 0 & 1 & 10 & 100 \\
\hline & 1 & $2.29110^{-1}$ & $8.30010^{-2}$ & $1.88810^{-2}$ & $9.24310^{-4}$ \\
& 3 & $2.28710^{-1}$ & $8.35010^{-2}$ & $1.89010^{-2}$ & $9.24210^{-4}$ \\
$\mathrm{~L}$ & 5 & $2.28210^{-1}$ & $8.35710^{-2}$ & $1.89010^{-2}$ & $9.24210^{-4}$ \\
& 7 & $2.28010^{-1}$ & $8.35910^{-2}$ & $1.89110^{-2}$ & $9.24210^{-4}$ \\
& $\infty$ & $2.27710^{-1}$ & $8.36210^{-2}$ & $1.89110^{-2}$ & $9.24210^{-4}$
\end{tabular}

\begin{tabular}{cccccc}
\hline \multirow{2}{*}{$\boldsymbol{T}=\mathbf{1 0}$} & \multicolumn{5}{c}{$u$} \\
\cline { 3 - 6 } & & 0 & 1 & 10 & 100 \\
\hline \multirow{2}{*}{$\mathrm{L}$} & $\mathbf{2}$ & $5.09010^{-1}$ & $3.78610^{-1}$ & $1.37910^{-1}$ & $9.36210^{-3}$ \\
& 3 & $5.13710^{-1}$ & $3.86010^{-1}$ & $1.40210^{-1}$ & $9.35310^{-3}$ \\
& 7 & $5.14410^{-1}$ & $3.86910^{-1}$ & $1.40610^{-1}$ & $9.35210^{-3}$ \\
& $\infty$ & $5.14610^{-1}$ & $3.87210^{-1}$ & $1.40710^{-1}$ & $9.35110^{-3}$ \\
& $\infty$ & $5.14810^{-1}$ & $3.87410^{-1}$ & $1.40810^{-1}$ & $9.35110^{-3}$
\end{tabular}

\begin{tabular}{cccccc}
\hline \multirow{2}{*}{$T=100$} & \multicolumn{5}{c}{$u$} \\
\cline { 2 - 6 } & & 0 & 1 & 10 & 100 \\
\hline & 1 & $7.45610^{-1}$ & $6.68410^{-1}$ & $4.36010^{-1}$ & $8.54210^{-2}$ \\
& 3 & $7.39210^{-1}$ & $6.62410^{-1}$ & $4.38710^{-1}$ & $8.61210^{-2}$ \\
$\mathrm{~L}$ & 5 & $7.38110^{-1}$ & $6.61310^{-1}$ & $4.38610^{-1}$ & $8.62310^{-2}$ \\
& 7 & $7.37810^{-1}$ & $6.60910^{-1}$ & $4.38610^{-1}$ & $8.62810^{-2}$ \\
& $\infty$ & $7.37510^{-1}$ & $6.60510^{-1}$ & $4.38410^{-1}$ & $8.63210^{-2}$ \\
\hline
\end{tabular}




\begin{tabular}{|c|c|c|c|c|c|}
\hline \multirow{2}{*}{$T=1004$} & & \multicolumn{4}{|c|}{$u$} \\
\hline & & 0 & 1 & 10 & 100 \\
\hline \multirow{5}{*}{$\mathbf{L}$} & 1 & $8.72810^{-1}$ & $8.34510^{-1}$ & $7.13610^{-1}$ & $3.55110^{-1}$ \\
\hline & 3 & $8.68110^{-1}$ & $8.29010^{-1}$ & $7.09410^{-1}$ & $3.61010^{-1}$ \\
\hline & 5 & $8.67610^{-1}$ & $8.28310^{-1}$ & $7.08510^{-1}$ & $3.61610^{-1}$ \\
\hline & 7 & $8.67410^{-1}$ & $8.28110^{-1}$ & $7.08110^{-1}$ & $3.61710^{-1}$ \\
\hline & $\infty$ & $8.67210^{-1}$ & $8.27810^{-}$ & $7.07710^{-1}$ & $3.61810^{-1}$ \\
\hline
\end{tabular}

As expected, the extrapolation clearly improves the convergence, yielding at least three relative significant digits in most cases. Of course, for practical purposes, most often even just one or two significant digits will suffice.

The same conclusions are apparent from the extrapolated approximations for the Erlang(3) claim sizes:

\begin{tabular}{|c|c|c|c|c|}
\hline \multicolumn{5}{|c|}{ Improved Erlang Approximations $\psi_{1}\left(u, H_{L}\right)$ to finite time ruin probabilities } \\
\hline & & Claim Size $f(x$ & Erlang(3)) & \\
\hline \multirow{2}{*}{$T=1$} & & \multicolumn{3}{|c|}{$u$} \\
\hline & & 0 & 1 & 10 \\
\hline \multirow{5}{*}{$\mathbf{L}$} & 1 & $5.13910^{-1}$ & $2.45110^{-1}$ & $-9.96310^{-6}$ \\
\hline & 3 & $5.28510^{-1}$ & $2.49610^{-1}$ & $5.00810^{-7}$ \\
\hline & 5 & $5.30810^{-1}$ & $2.50310^{-1}$ & $2.05710^{-6}$ \\
\hline & 7 & $5.31510^{-1}$ & $2.50510^{-1}$ & $2.55710^{-6}$ \\
\hline & $\infty$ & $5.32310^{-1}$ & $2.50810^{-1}$ & $3.14610^{-6}$ \\
\hline \multirow{2}{*}{$r=10$} & & \multicolumn{3}{|c|}{$u$} \\
\hline & & 0 & 1 & 10 \\
\hline \multirow{5}{*}{$\mathrm{L}$} & 1 & $8.28910^{-1}$ & $6.14510^{-1}$ & $1.02910^{-2}$ \\
\hline & 3 & $8.17610^{-1}$ & $6.15510^{-1}$ & $9.22410^{-3}$ \\
\hline & 5 & $8.15810^{-1}$ & $6.14810^{-1}$ & $8.99910^{-3}$ \\
\hline & 7 & $8.15310^{-1}$ & $6.14510^{-1}$ & $8.91410^{-3}$ \\
\hline & $\infty$ & $8.14810^{-1}$ & $6.14210^{-1}$ & $8.79710^{-3}$ \\
\hline \multirow{2}{*}{$T=100$} & & \multicolumn{3}{|c|}{$u$} \\
\hline & & 0 & 1 & 10 \\
\hline \multirow{5}{*}{$\mathrm{L}$} & 1 & $9.06410^{-1}$ & $7.90410^{-1}$ & $1.53310^{-1}$ \\
\hline & 3 & $8.98310^{-1}$ & $7.81110^{-1}$ & $1.59510^{-1}$ \\
\hline & 5 & $8.97610^{-1}$ & $7.80010^{-1}$ & $1.60410^{-1}$ \\
\hline & 7 & $8.97510^{-1}$ & $7.79710^{-1}$ & $1.60610^{-1}$ \\
\hline & $\infty$ & $8.97310^{-1}$ & $7.79310^{-1}$ & $1.60810^{-1}$ \\
\hline
\end{tabular}




\begin{tabular}{cccccc}
\hline \multirow{2}{*}{$\boldsymbol{T}=\mathbf{1 0 0 0}$} & \multicolumn{5}{c}{$u$} \\
\cline { 2 - 6 } & & 0 & 1 & 10 & 100 \\
\hline \multirow{4}{*}{$\mathbf{L}$} & 1 & $9.12510^{-1}$ & $8.10710^{-1}$ & $2.38310^{-1}$ & $4.75910^{-7}$ \\
& 3 & $9.09310^{-1}$ & $8.05010^{-1}$ & $2.32610^{-1}$ & $5.21410^{-7}$ \\
& 5 & $9.09210^{-1}$ & $8.04510^{-1}$ & $2.31610^{-1}$ & $5.40010^{-7}$ \\
& 7 & $9.09110^{-1}$ & $8.04410^{-1}$ & $2.31310^{-1}$ & $5.50110^{-7}$ \\
& $\infty$ & $9.09110^{-1}$ & $8.04310^{-1}$ & $2.31010^{-1}$ & $5.73710^{-7}$ \\
\hline \hline
\end{tabular}

\section{REFERENCES}

1. Aldous, D. and Shepp, L. (1987) The least variable phase-type distribution is Erlang. Stochastic Models 3, 467-473.

2. ASMUSSEN, S. (1989) Risk theory in a Markovian environment. Scand. Act. J. 89, 69-100.

3. ASMusSEN, S. (1992) Phase-type representations in random walk and queueing problems. Ann. Probab. 20, 772-789.

4. Asmussen, S. (1995a) Stationary distributions for fluid flow models with or without Brownian noise. Stochastic Models 11, 21-49.

5. ASMUSSEN, S. (1995b) Stationary distributions via first passage times. Advances in Queueing (J. Dshalalow, ed.), 79-102. CRC Press.

6. Asmussen, S. (2000) Matrix-analytic models and their analysis. Scand. J. Statist. 27, 193-226.

7. ASMUSSEN, S. (2000) Ruin Probabilities. World Scientific.

8. ASMUSSEN, S. and BLADT, M. (1996) Renewal theory and queueing algorithms for matrixexponential distributions. Matrix-Analytic Methods in Stochastic Models (A.S. Alfa \& S. Chakravarty, eds.), 313-341. Marcel Dekker, New York.

9. Asmussen, S. and Kella, O. (2000) A multi-dimensional martingale for Markov additive processes and its applications. Adv. Appl. Probab. 32, 376-393.

10. Asmussen, S. and Rolski, T. (1991) Computational methods in risk theory: a matrix-algorithmic approach. Insurance: Mathematics and Economics 10, 259-274.

11. Avram, F. and Usabel, M. (2001) Finite time ruin probabilities with one Laplace inversion. Submitted to Insurance: Mathematics and Economics.

12. AVRAm, F. and UsabeL, M. (2001) Ruin probabilities and deficit for the renewal risk model with phase-type interarrival times. Submitted to Scand. Act. $J .19$.

13. Avram, F. and Usabel, M. (2002) An ordinary differential equations approach for finite time ruin probabilities, including interest rates. Submitted to Insurance: Mathematics and Economics.

14. BARLOW, M.T., Rogers, L.C.G. and Williams, D. (1980) Wiener-Hopf factorization for matrices, in Seminaire de Probabilites XIV, Lecture Notes in Math. 784, Springer, Berlin, 324-331.

15. Gerber, H., GoovaERTS, M. and KAAS, R. (1987) On the probability and severity of ruin. Astin Bulletin 17, 151-163.

16. GraHAM, A. (1981) Kronecker Products and Matrix Calculus. Wiley. \%

17. Latouche, G. and Ramaswami, V. (1999) Introduction to Matrix-Analytic Methods in Stochstic Modelling. SIAM.

18. London, R.R., MCKeAN, H.P., Rogers, L.C.G. and Williams, D. (1982) A martingale approach to some Wiener-Hopf problems II, in Seminaire de Probabilités XVI, Lecture Notes in Math. 920, Springer, Berlin, 68-90.

19. Mitra, D. (1988), Stochastic Fluid Models, Performance 87, P. J. Courtois and G. Latouche (editors), Elsevier, (North-Holland), 39-51.

20. Neuts, M.F. (1981) Matrix-Geometric Solutions in Stochastic Models. Johns Hopkins University Press, Baltimore. London.

21. NeuTs, M.F. (1989) Structured Stochastic Matrices of the M/G/1 Type and Their Applications. Marcel Dekker. 
22. Press, W.H., Flannery, B.P., Teukolsky, S.A. and Vetterling, W.T. (1986) Numerical recipes. Cambridge University Press.

23. STANDFORD, D.A. and STROIŃSKI, K.J. (1994) Recursive method for computing finite-time ruin probabilities for phase-distributed claim sizes. Astin Bulletin 24, 235-254.

24. UsABEL, M. (1999) Calculating multivariate ruin probabilities via Gaver-Stehfest inversion technique. Insurance: Mathematics and Economics 25, 133-142.

25. WIKSTAD, N. (1971) Exemplification of ruin probabilities. Astin Bulletin 7, 147-152.

\section{SOREN ASMUSSEN}

Mathematical Statistics

Centre for Mathematical Sciences

Box 118, 22100 Lund, Sweden

E-mail: asmus@maths.lth.se

FLORIN AVRAM

Dept. de Math., Universite de Pau and Dept. of Actuarial Maths. \& Statistics Heriot-Watt University, Edinburgh EHI4 4 AS U.K.

E-mail: Florin.Avram@univ-Pau.fr

Miguel USABEL

Edif. Miguel de Unamuno, Universidad Carlos III

Avda. Universidad Carlos III 22, 28270 Colmenarejo (Madrid), Spain

E-mail: usabel@emp.uc3m.es 